\title{
Speech Enhancement for \\ Hands-Free Terminals
}

Nedelko Grbic,

Sven Nordholm and Anders Johansson 


\section{Contents}

- Handsfree Telephony Principles

- Handsfree problem

- Optimal Beamformers

- Linearly Constrained Minimum Variance Beamfomer

- Optimal Signal-to-Noise plus Interference

- Diffuse Noise Field Beamformer

- Minimum Mean Square Error Beamformer

- Results in a real environment

- Conclusions 


\section{Handsfree Telephony}

- Safety problems in cars

- Inconvenience of conversation

- Prohibited by legislation in some regions

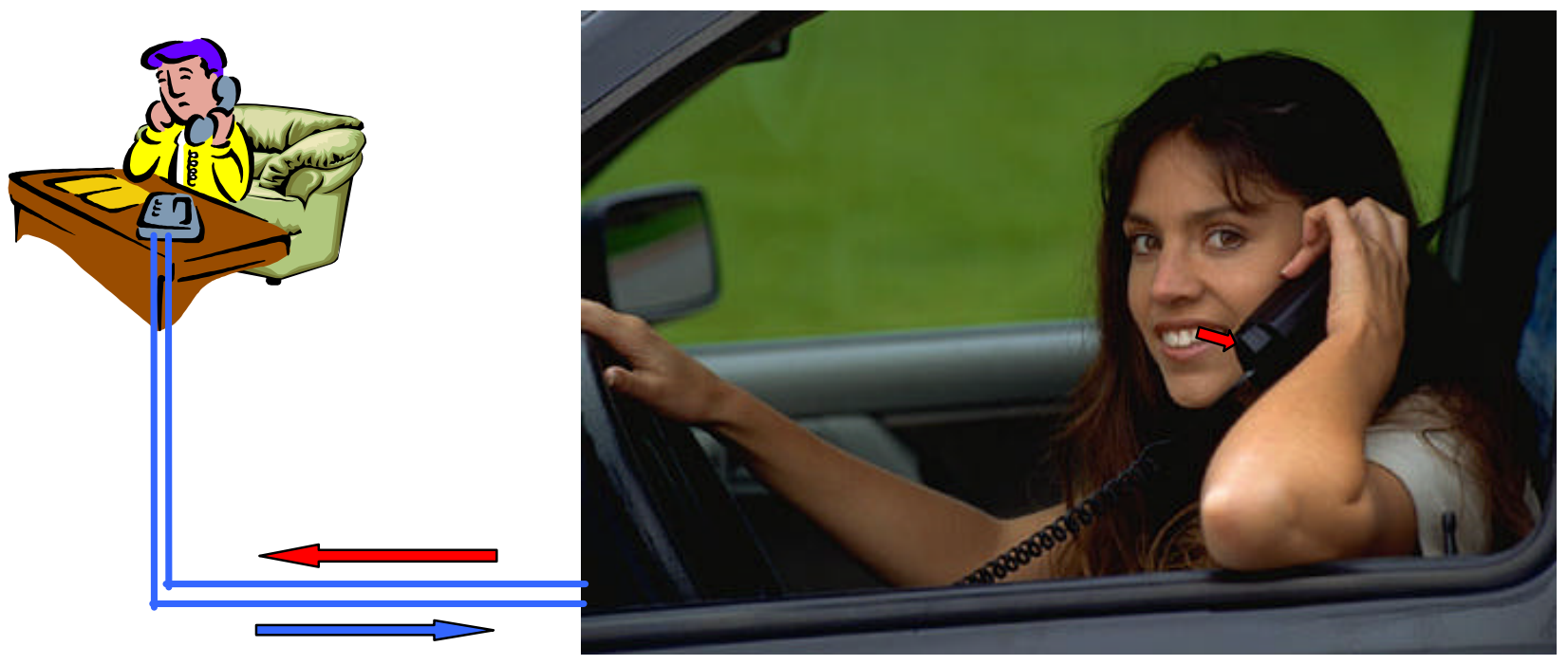

(C) Nedelko Grbic 


\section{Handsfree Telephony}

- Perception problems

- Acoustic feedback

- Wind and Tire friction in cars

- Engine and Fan noise

Single Mic.

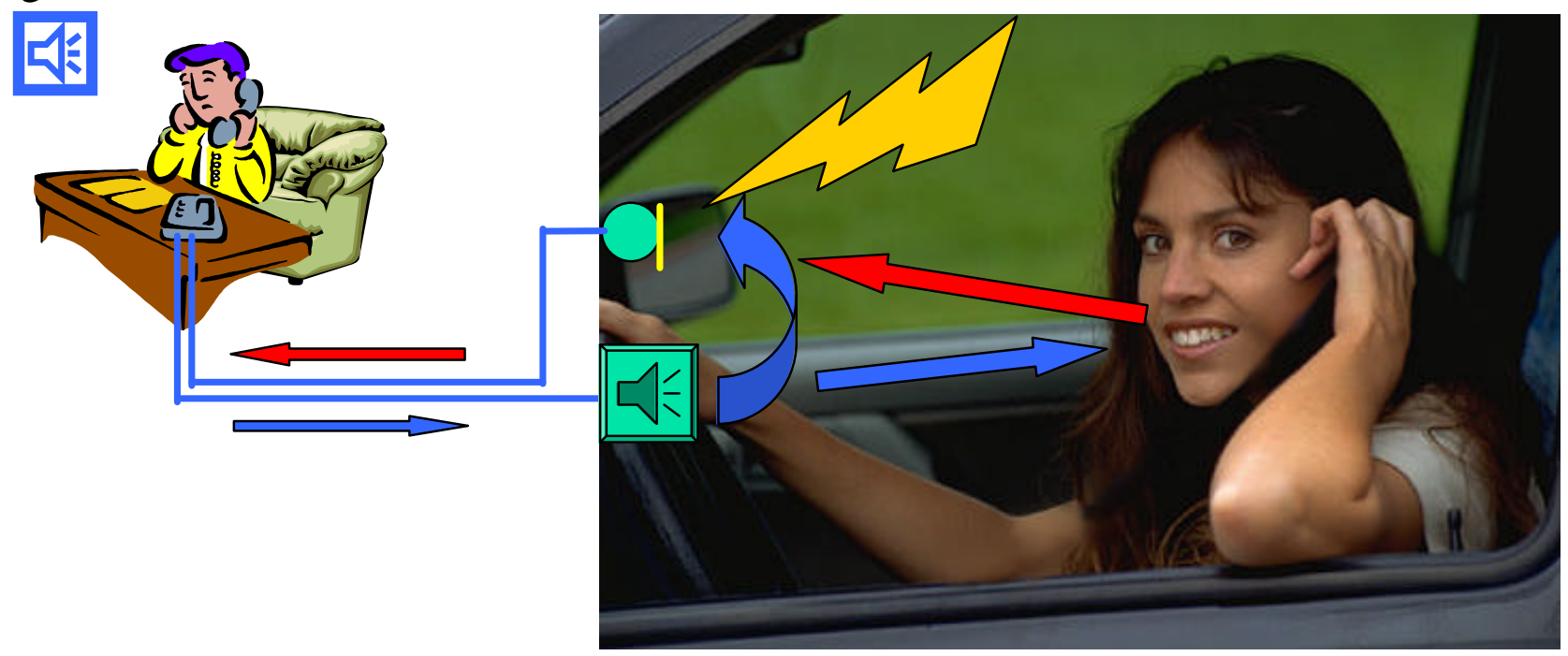

(C) Nedelko Grbic 


\section{Handsfree Telephony}

- Speech enhancement by means of beamforming

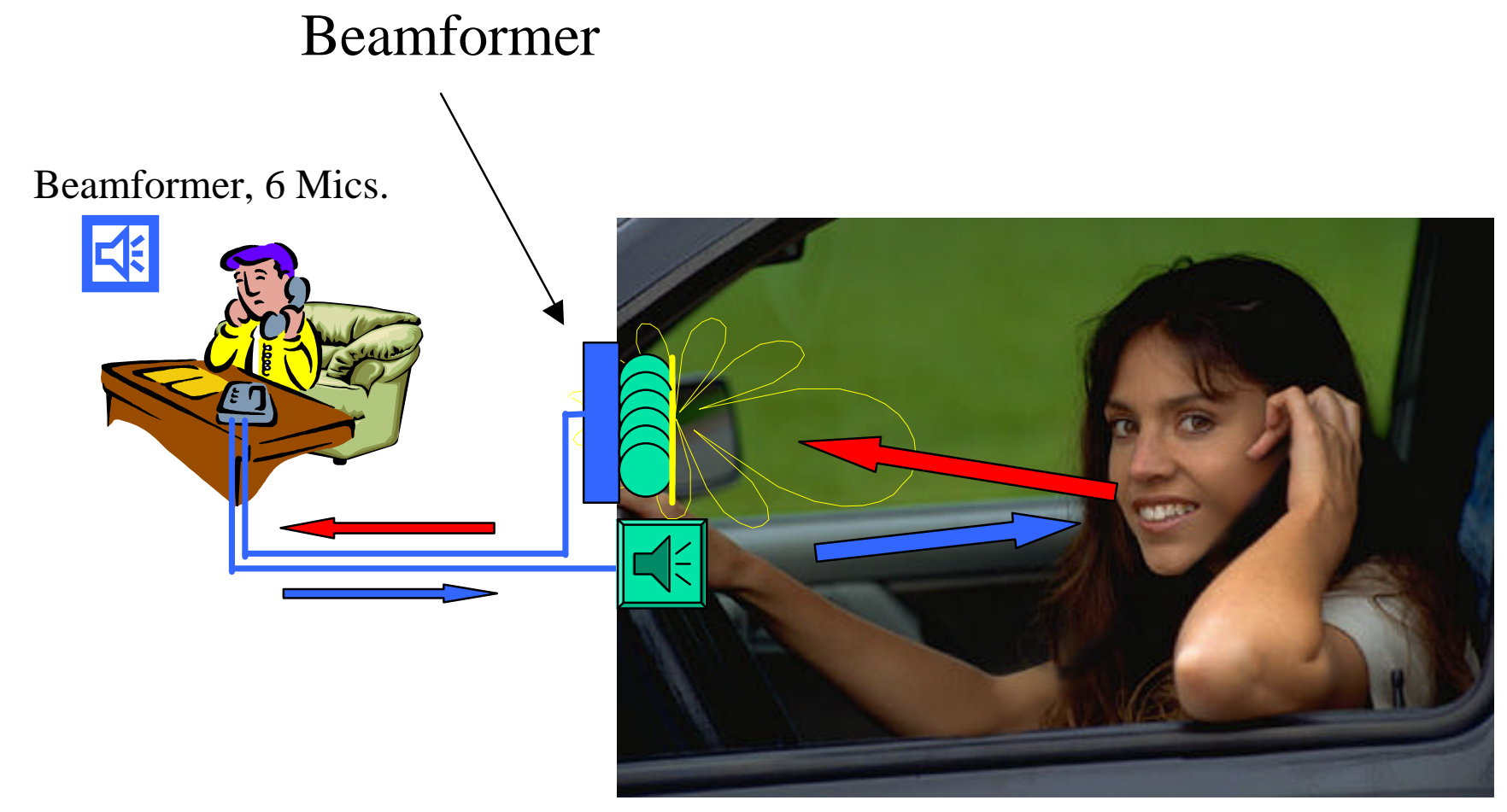

(C) Nedelko Grbic 


\section{Handsfree problem}
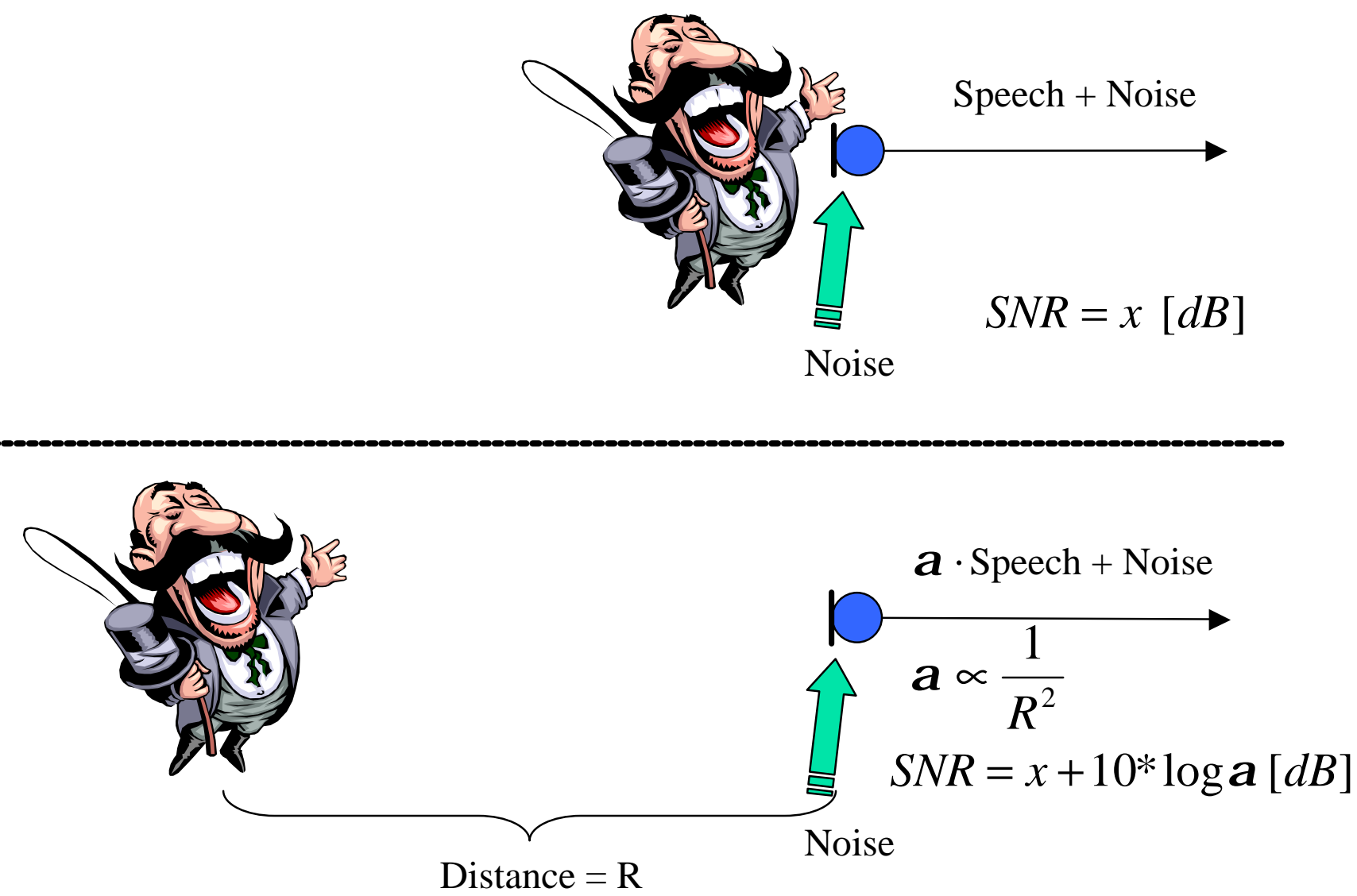

(C) Nedelko Grbic 


\section{Handsfree Improvement}

$$
S N R=x+10^{*} \log \left(\frac{\alpha}{\beta}\right)[d B]
$$
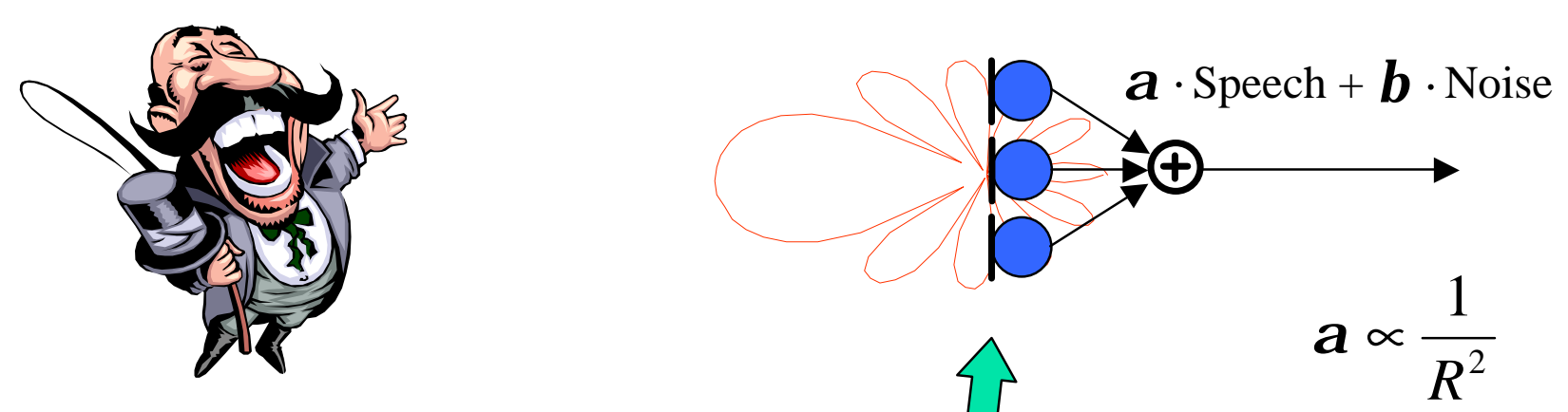

$\beta \propto \#$ Sensors

Noise

Distance $=\mathrm{R}$ 


\section{Spatial Selectivity}
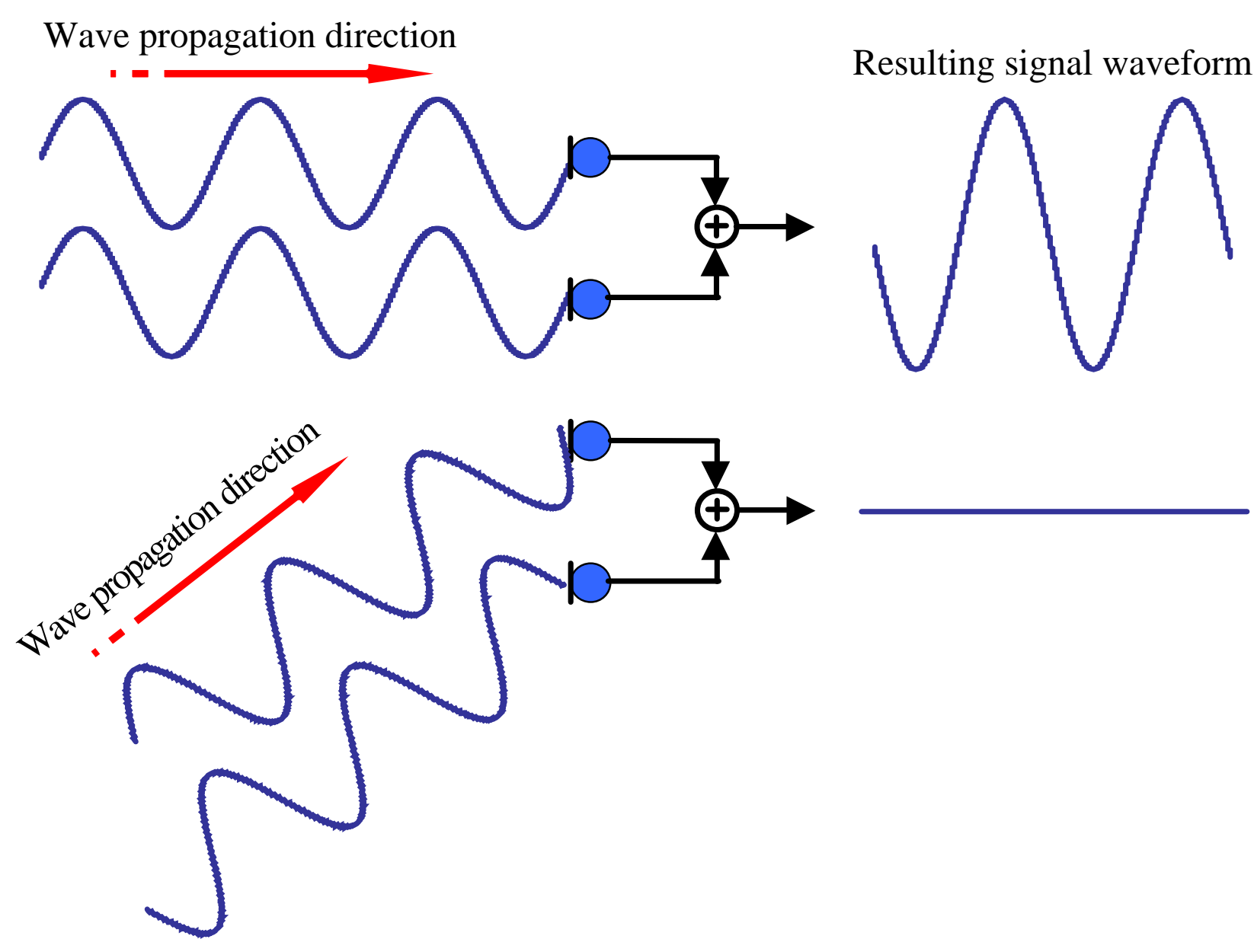

(C) Nedelko Grbic 


\section{Spatial Selectivity}
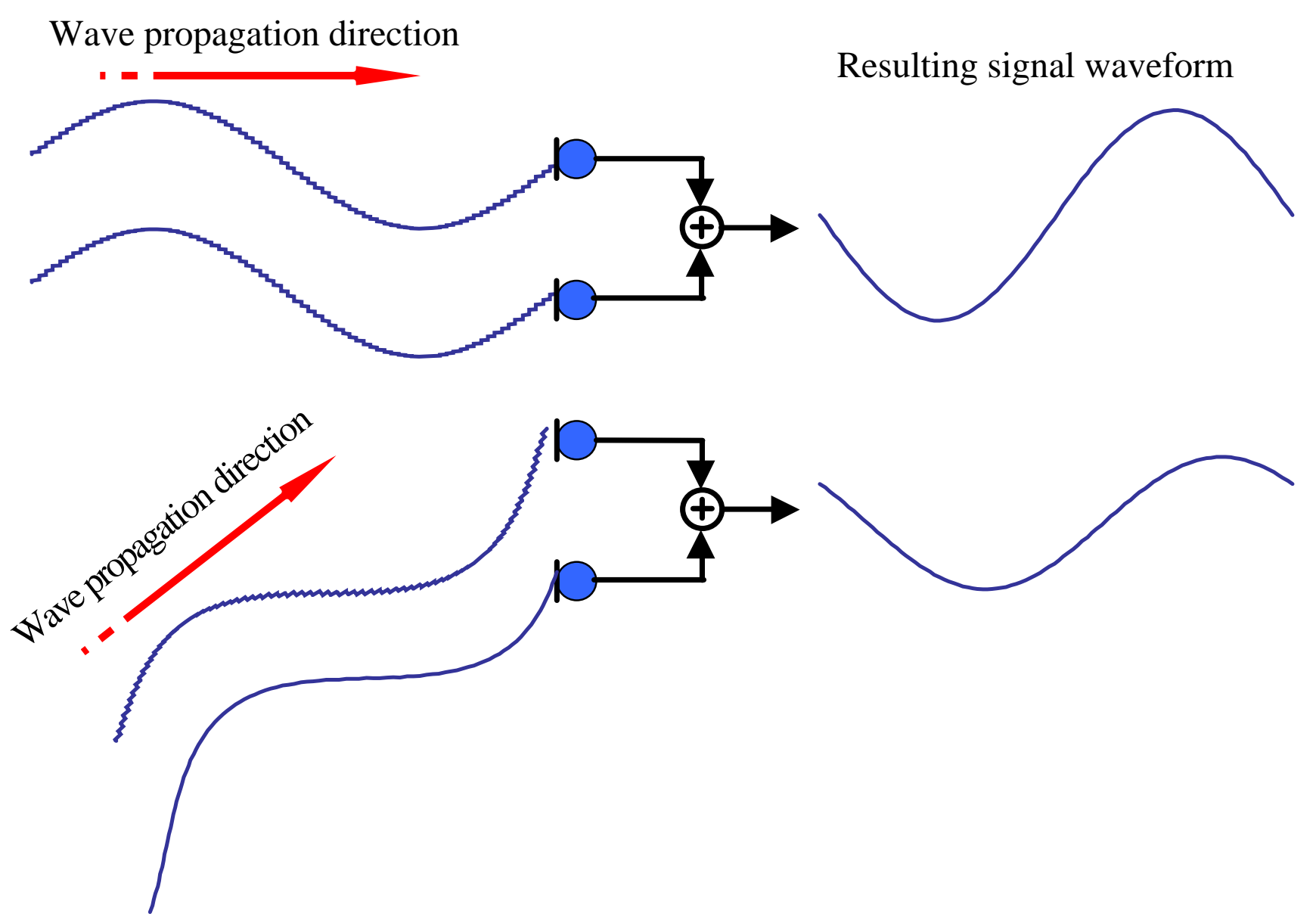

(C) Nedelko Grbic 


\section{Broadband Beamformer}

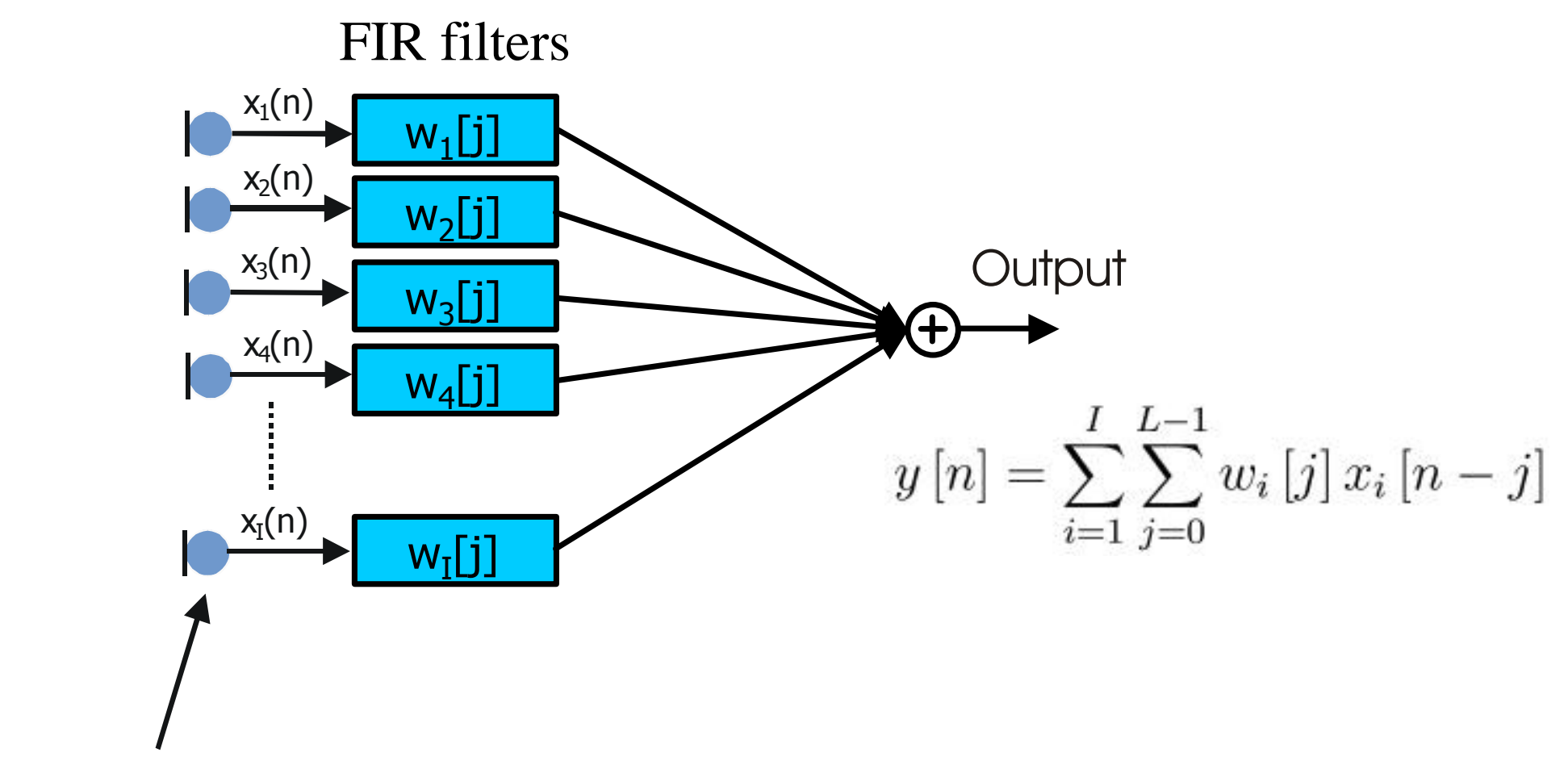

\#I Microphones 


\section{Ex. Broadband response}

Frequency-Spatial Amplitude response, \#Sensors = 10, Radius = 10, Sensor dist. $=0.05$

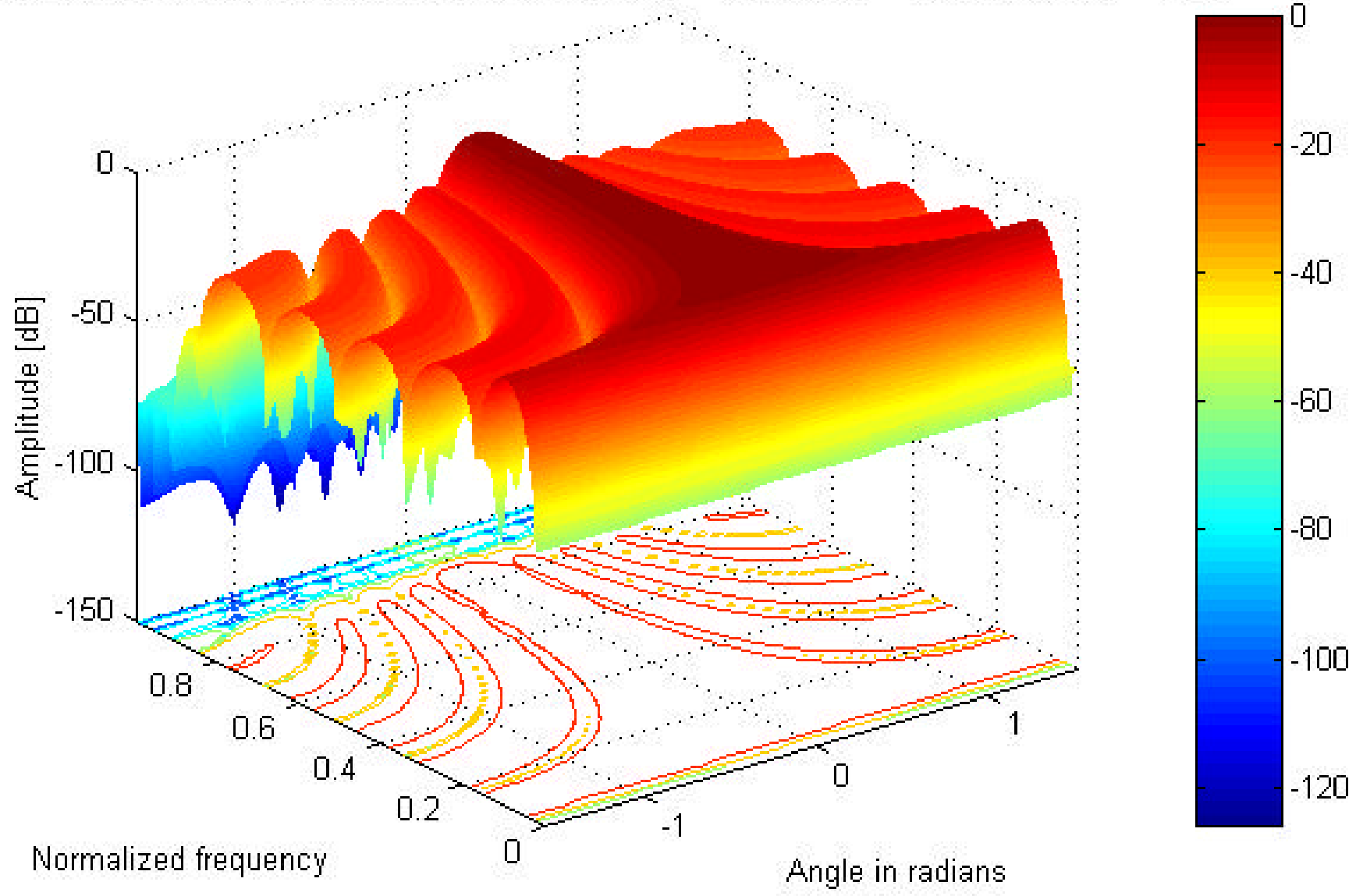

(C) Nedelko Grbic 


\section{Beamforming approaches}

\section{Data independent Beamformers}

- The Delay and Sum Beamformer

- Multidimensional Filter designed Beamformers

Statistical Beamformers

- Linearly Constrained Minimum Variance Beamforming

- The Optimal Signal-to-Noise plus Interference (SNIB) Beamformer

- Minimum Mean Square Beamformer

- Diffuse Noise Field Beamformer 


\section{Linearly Constrained Minimum Variance Beamformer (LCMV)}

For each frequency, the weights are found

from:

$$
\begin{gathered}
\left.\begin{array}{c}
\min _{\mathbf{w}} \mathbf{w}^{H} \mathbf{R}_{\mathbf{x x}}(\Omega) \mathbf{w} \\
\text { Subject to: } \\
\mathbf{d}^{H}(R, \theta, \Omega) \mathbf{w}=g^{*}
\end{array}\right\} \Rightarrow \\
\Rightarrow \quad \mathbf{w}_{\text {opt }}=g^{*} \frac{\mathbf{R}_{\mathbf{x x}}^{-1}(\Omega) \mathbf{d}(R, \theta, \Omega)}{\mathbf{d}^{H}(R, \theta, \Omega) \mathbf{R}_{\mathbf{x x}}^{-1}(\Omega) \mathbf{d}(R, \theta, \Omega)}
\end{gathered}
$$

where $\mathbf{d}(R, \theta, \Omega)=\left[\frac{1}{R}, \frac{1}{R_{1}} e^{-j \Omega \tau_{1}(R, \theta)}, \ldots, \frac{1}{R_{L-1}} e^{-j \Omega \tau_{L-1}(R, \theta)}\right]$

The correlation matrix $\mathbf{R}_{\mathbf{x x}}(\Omega)$ contains contributions from all sources

If $\mathrm{g}=1$, then LCMV equals the Minimum Variance Distortionless Response, (MVDR)

(C) Nedelko Grbic 


\section{Optimal SNIB Beamformer}

\section{$Q=\frac{\text { average signal output power }}{\text { average }}$ \\ average noise-plus-interference output power}

$$
\mathbf{w}_{\text {opt }}=\arg \max _{\mathbf{w}} \frac{\mathbf{w}^{H} \mathbf{R}_{\mathbf{s s}} \mathbf{w}}{\mathbf{w}^{H} \mathbf{R}_{\mathbf{n} \mathbf{w}} \mathbf{w}}
$$

The correlation matrix $\mathbf{R}_{\mathrm{ss}}$ contains contributions from the source of interest and $\mathbf{R}_{\mathbf{n n}}$ contains contributions from all other sources

The weights that maximizes the quote, are found from the Generalized Eigenvalue relation, i.e.,

$$
\begin{gathered}
\mathbf{w}_{\text {opt }}=\arg \max _{\mathbf{w}}\left\{\frac{\mathbf{w}^{H} \mathbf{R}_{\mathbf{s s}} \mathbf{w}}{\mathbf{w}^{H} \mathbf{R}_{\mathbf{n n}} \mathbf{w}}\right\} \Rightarrow \mathbf{R}_{\mathbf{s s}} \mathbf{w}_{\text {opt }}=\lambda_{\max } \mathbf{R}_{\mathbf{n n}} \mathbf{w}_{\text {opt }} \\
\Rightarrow>\mathbf{R}_{\mathbf{n n}}^{-1} \mathbf{R}_{\mathbf{s s}} \mathbf{w}_{\text {opt }}=\lambda_{\max } \mathbf{w}_{\text {opt }} \mid
\end{gathered}
$$

(C) Nedelko Grbic 


\section{MMSE Beamformer}

$$
\begin{gathered}
\mathbf{w}_{\text {opt }}=\arg \min _{\mathbf{w}} E\left\{\left|y[n]-s_{r}[n]\right|^{2}\right\} \quad r \in[1,2, \cdots, I] \mid \\
\mathbf{w}_{\text {opt }}=\arg \min _{\mathbf{w}}\left\{E\left[\left|\mathbf{w}^{H} \mathbf{s}[n]-s_{r}[n]\right|^{2}+\left|\mathbf{w}^{H} \mathbf{x}[n]\right|^{2}\right]\right\} \\
\mathbf{w}_{\text {opt }}=\arg \min _{\mathbf{w}}\left\{\mathbf{w}^{H}\left[\mathbf{R}_{\mathbf{s s}}+\mathbf{R}_{\mathbf{x x}}\right] \mathbf{w}-\mathbf{w}^{H} \mathbf{r}_{s}-\mathbf{r}_{\mathbf{s}}^{\mathbf{H}} \mathbf{w}+r_{s_{r}}\right\} \\
\mathbf{w}_{\text {opt }}=\left[\mathbf{R}_{\mathbf{s} \mathbf{s}}+\mathbf{R}_{\mathbf{x x}}\right]^{-1} \mathbf{r}_{\mathbf{s}}
\end{gathered}
$$




\section{Diffuse Noise Field beamformer}

For each frequency, the weights are found

from:

$$
\mathbf{w}_{\text {opt }}=\mathbf{R}_{\mathbf{n n}}^{-1}(\Omega) \mathbf{d}(R, \theta, \Omega)
$$

where

$$
\mathbf{d}(R, \theta, \Omega)=\left[\frac{1}{R}, \frac{1}{R_{1}} e^{-j \Omega \tau_{1}(R, \theta)}, \ldots, \frac{1}{R_{L-1}} e^{-j \Omega \tau_{L-1}(R, \theta)}\right]
$$

and

$$
\mathbf{R}_{n_{i} n_{j}}(\Omega)=\sigma^{2} \frac{\sin \left(k d_{i j}\right)}{k d_{i j}}
$$




\section{Evaluation Conditions}

- Environment in car running at $110 \mathrm{~km} / \mathrm{h}$

- Linear sensor array

- 6 sensors with $12 \mathrm{kHz}$ sampling rate

- Evaluation on real speech signals 


\section{Results}

\begin{tabular}{|l|c|c|c|}
\hline Performance $[\mathrm{dB}]$ & Speech Distortion & Noise Suppression & Interference Suppression \\
\hline SNIB & -19.4 & 18.1 & 30.7 \\
\hline MMSE & -30.6 & 15.2 & 17.2 \\
\hline Diffuse Noise Field & -26.5 & 4.0 & 1.9 \\
\hline
\end{tabular}
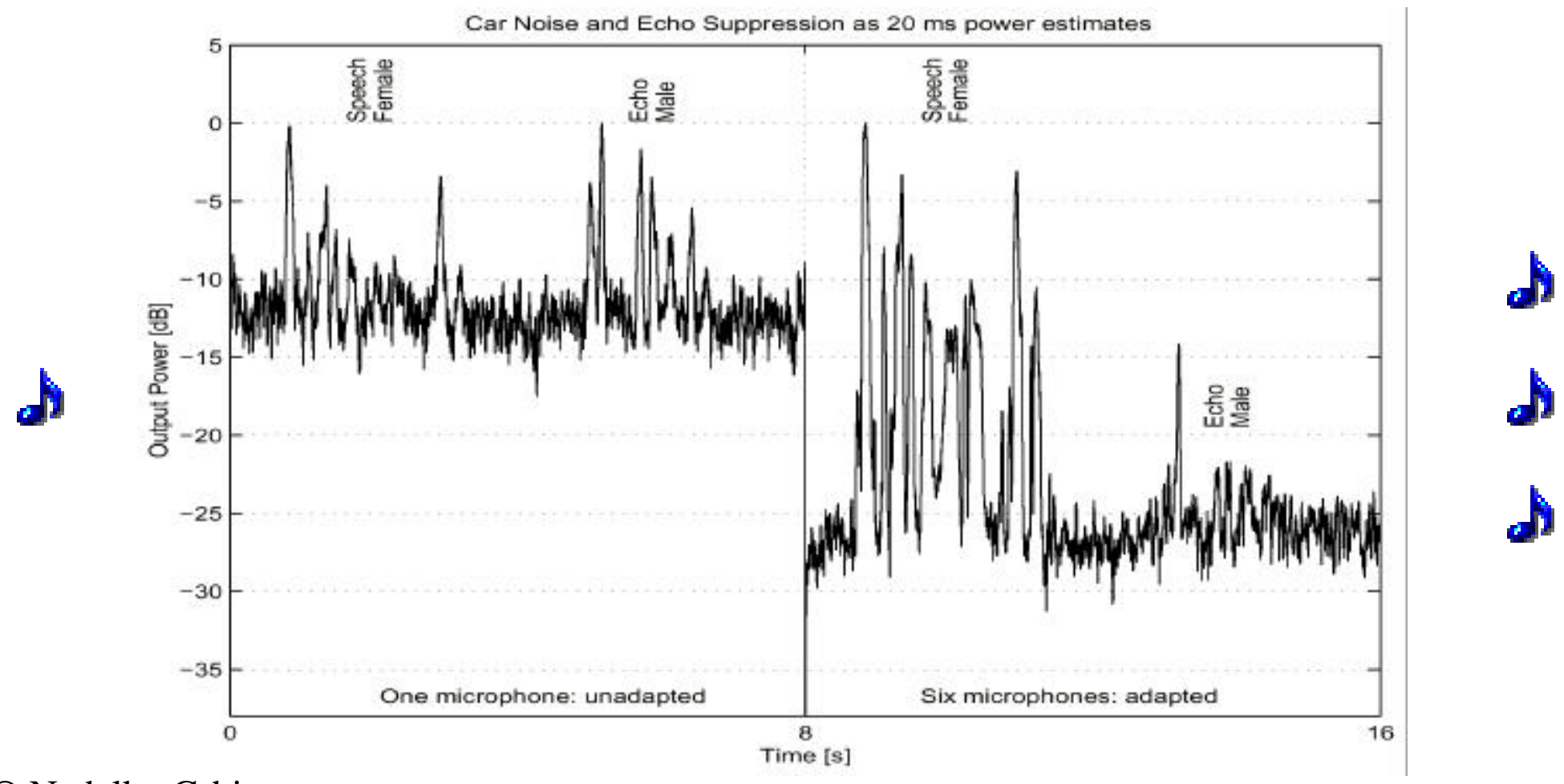

(C) Nedelko Grbic 


\section{Conclusions}

- Multisensor techniques are efficient in a terminal handsfree situation

- An SNR improvement of 15-18 dB can be achieved with six sensors

- The SNIB has better noise suppression than the MMSE but also more distortion

- The Diffuse Noise field model is inaccurate in a car handsfree environment 